\title{
CARCINOMA INVASIVO Y EMBARAZO
}

\section{Experiencia de 11 años consecutivos}

\author{
Prof. Rubén Darío Guzmán A.* \\ Prof. Alvaro Fonnegra M.**
}

Dilucidar si el embarazo ejerce un efecto definitivo sobre el carcinoma del cuello uterino es tema lleno de controversias y de opiniones no precisas. Lo que sí parece cierto, como lo afirman Marsh y Frigerald (1), Kistner (2) y Kinch (3), es que no existen datos definitivos que indiquen que el carcinoma del cervix experimente diferenciación o acelere su capacidad invasora bajo la influencia del embarazo o de las hormonas. No obstante, es aceptado por todos que cuando el producto de la concepción se expulsa a través del cuello canceroso se acelera el crecimiento tumoral y por lo tanto disminuye la supervivencia de la enferma embarazada. Sin embargo, autores como J. León (4) consideran que la influencia del embarazo sobre el cáncer del cuello uterino es bastante perjudicial, sobre todo en la mujer joven; esto ha sido atribuído a la intensa vascularización, al aumento del glucógeno en los genitales y a la elevada concentración de las gonadotrofinas y de los estrógenos. Por fin, algunos más optimistas, piensan que la gravidez retarda la evolución del carcinoma. Lo cierto es que en estos últimos años parece aceptado que en la mayoría de las pacientes dicha influencia no es tan desfavorable, lo que no constituye

un motivo para que el médico no tenga que prestar especial atención a su diagnóstico y tratamiento precoz; igual opinión comparte Emge (5). En realidad, el ritmo de crecimiento parece ser inherente a la misma neoplasia; la aceleración aparente del desarrollo de algunos carcinomas durante el embarazo quizá guarde relación más importante con la edad, la paridad etc. Waldrep y Palmer (6) sostienen que excepcionalmente el embarazo influye en el crecimiento tumoral. Lo que también parece cierto es la mala influencia de la afección sobre el embarazo, pudiendo producir abortos, partos prematuros, inserción baja de la placenta, fetos de bajo peso etc.

\section{Material y Método}

Se estudian y analizan 37 casos de carcinoma invasivo de cervix uterino concomitante con embarazo en un lapso de 11 años en el Instituto Materno Infantil "Concepción Villaveces de Acosta" de Bogotá, del 10 de Enero de 1960 hasta el 30 de Septiembre de 1971.

* Profesor Asistente, Depto. Obstetricia y Ginecología, Facultad de Medicina U.N.

** Profesor Asociado, Depto. Obstetricia y Ginecología, Facultad de Medicina U.N. 


\section{Frecuencia}

Es muy variada, pues algunas instituciones son puramente obstétricas y la relación se obtiene con el número de pacientes embarazadas atendidas; otras están enteramnete dedicadas a la atención de tumores malignos, y la relación se obtiene con el número de pacientes con carcinoma del cervix.

Según J. León (4), la frecuencia oscila entre $0,01 \%$ y $0,1 \%$. En Chicago Lying In Hospital, según Wilson, ocurrió una vez en cada 6.620 partos. En el Instituto Materno Infantil de Bogotá, la frecuencia según Arévalo y Lozano (8) es de 0,031\%. En general la frecuencia es de una por cada 2.000 embarazadas (9).

\section{Paridad}

Multíparas: 37

$(100 \%)$

Sostienen Kistner y Gorbach que en cualquier serie de casos de carcinoma de cuello uterino se eleva la frecuencia en las multíparas, sobre todo después del $44^{\circ}$ o $5^{\circ}$ embarazo. Ello ha hecho que varios observadores como Waugh y Pike (10) afirmen que la infección cervical secundaria a desgarros en el parto es factor carcinógeno, por lo que puede atribuirse un papel predisponente a las erosiones, eversiones y laceraciones, tan frecuentes en ellos.

\section{Edad}

Menores de 25 años:

Entre 26 y 35 años:

Entre 36 y 45 años:

\begin{tabular}{rr}
1 & $3 \%$ \\
23 & $62 \%$ \\
13 & $35 \%$ \\
\hline 37 & $100 \%$
\end{tabular}

La mayor incidencia ocurrió en las pacientes entre los 26 y 35 años.

\section{Estado del cuello uterino}

Gran número de las enfermas presentaban erosiones, ulceraciones de toda índole, masas polipoides etc. que no detallamos por carecer de importancia.

\section{Motivo de Consulta}

Fueron semejantes a las de cualquier proceso maligno (Flujo, hemorragia genital, dolor etc.), más los propios del estado grávido.

\section{Embarazo en semanas}

$\begin{array}{lllrr}\text { Entre la 5a. y la 10a. } & =5 & 13 \% \\ \text { Entre la 11a. y la 15a. } & =5 & 13 \% \\ \text { Entre la 16a. y la 20a. } & =5 & 13 \% \\ \text { Entre la 21a. y la 25a. } & =1 & 3 \% \\ \text { Entre la 26a. y la 30a. } & =3 & 9 \% \\ \text { Entre la 31a. y la 35a. } & =1 & 3 \% \\ \text { Entre la 36a. y la 40a. } & =15 & 40 \% \\ \text { Puérperas } & & 2 & 6= \\ & & & 37 & \\ \end{array}$

La mayor frecuencia de carcinoma de cervix ocurrió entre la 36: y la 40 a semanas de gestación.

\section{Estados clínicos}

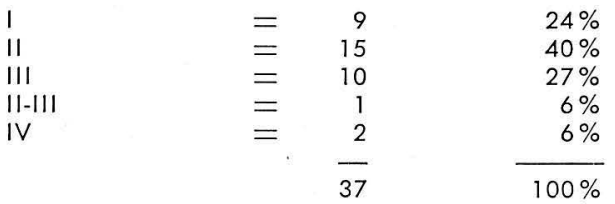

La mayor incidencia correspondió a los estados II y III. Desafortunadamente, nuestras pacientes llegan en estado avanzado cuando el pronóstico es bastante incierto. Esperamos que con una buena consulta prenatal y con citología vaginal rutinaria, la pesquisa del carcinoma del cérvix en el estado 0 sea el más frecuente y así podamos salvar la vida de nuestras enfermas, ya que todos sabemos que en este estado el índice de curación se acerca al $100 \%$.

\section{Citología vaginal}

\begin{tabular}{llrr} 
A3 & $=2$ & $6 \%$ \\
A4 & $=6$ & $17 \%$ \\
A5 & 2 & $6 \%$ \\
No se efectuó & $=27$ & $71 \%$ \\
\cline { 2 - 3 } & & $\frac{1}{37}$ &
\end{tabular}


Criticable es la deficiencia en no haber tomado 27 citologías vaginales en estas pacientes; sin embargo, es bueno aclarar que en nuestra institución este procedimiento se realiza de manera rutinaria desde hace aproximadamente seis años y la mayoría de los casos ocurrieron antes, cuando la citología exfoliativa era un método de rara aplicación. Es imperativo recalcar, como dice Sadugor, citado por J. León (4), la importancia de esta consulta ya que el diagnóstico precoz del carcinoma del cérvix depende en muchas ocasiones del médico, pues las manifestaciones clínicas con que suelen consultar estas pacientes son interpretadas erróneamente; así, las hemorragias indoloras que se observan en el $73 \%$ de las enfermas son atribuídas en el primer trimestre a una amenaza de aborto o a un embarazo ectópico; en el último trimestre, a placenta previa o desprendimiento prematuro de placenta. Lo mismo ocurre con las secreciones vaginales sanguinolentas, acuosas o purulentas, que se presentan en el $19 \%$ de los casos y que suelen achacarse a tricomoniasis vaginal, moniliasis, etc. Otro motivo para no hacer en la gestante diagnóstico de $\mathrm{Ca}$ de cuello uterino, es que se eluden en ellas el tacto vaginal, la inspección del cuello con espéculo y la citología vaginal.

\section{Biopsia del cuello uterino}

Carcinoma In situ

Ca escamocelular

\begin{tabular}{rr}
1 & $3 \%$ \\
36 & $97 \%$ \\
\hline 37 & $100 \%$
\end{tabular}

El primer caso $(\mathrm{Cl})$ corresponde a enferma a quien se le hizo el diagnóstico de ca de cuello estado I; la biopsia informó un $\mathrm{Ca}$ in situ; se le conizó el cuello uterino con objeto de aclarar el diagnóstico; el resultado fue Ca invasivo. Posteriormente se sometió a histerectomía radical, falle- ciendo en el acto quirúrgico por hemorragia por afibrinogenemia.

\section{Traĩamiento y estados clínicos}

Cesárea con histerectomía radical, Estado I

Cesárea corporal, estado II-III

Cesárea corporal, estado II

Cesárea corporal, estado III

Cesárea corporal, estado IV

Histerectomía Radical, estado I

Histerectomía Radical, estado II

Histerectomía Abdominal Total con feto in utero, estado III

Histerectomía Abdominal Total, estado I

$$
\begin{array}{ll}
= & 3 \\
= & 1 \\
= & 6 \\
= & 5 \\
= & 1 \\
= & 3 \\
= & 2 \\
= & 1 \\
= & 1 \\
& 23
\end{array}
$$

Los demás casos se trataron por medio de revisión uterina o por medio de Raspado Uterino en caso de abortos enviándose posteriormente al INC $O$ se enviaron directamente al INC por considerarse su estado muy avanzado para ser intervenidos quirúrgicamente.

Nosotros seguimos las normas recomendadas por la mayoría de los autores, de tal manera que en el estado 1, 10 y 2 : trimestre, hacemos la histerectomía radical, haciendo caso omiso del embarazo y en el 3er. trimestre esperamos mayor viabilidad y practicamos cesárea corporal entre la 28 a y la 34 a semana de gestación, seguida de histerectomía radical. En los estados II, III, IV, ler. y 2 : trimestre: Roentgenoterapia profunda o cobaltoterapia y una vez aborte practicar Radiumterapia dos o cuatro semanas más tarde. Solamente se hará histerectomía abdominal, con el objeto de evacuar el útero en caso de no producirse el aborto después de la Roentgenoterapia o Cobaltoterapia. En el 3er. trimestre: Cesárea corporal tan pronto se considere viable el producto de la concepción (entre la 28: y la 34. semanas de la gestación). Se iniciará la terapéutica con roentgenoterapia profunda o cobaltoteraria 7 
a 10 días más tarde. Después de terminar la irradiación externa se administrará radioterapia.

En el cuadro anterior vemos que hubo conductas muy erróneas debidas a conceptos diversos y no muy claros, sobre el manejo de estas pacientes.

También son criticables 2 casos de parto por vías naturales, ya que aparte de la hemorragia que pueda producirse durante la dilatación cervical, la diseminación rápida de la enfermedad es lo corriente y por lo tanto el pronóstico se ensombrece.

Referente al promedio de curación según los diferentes estados del cuello uterino se ha discutido mucho, así, Waldrep y Palmer (6), consideran que los promedios de curación son muy semejantes en la primera y segunda mitad del embarazo. Sin embargo es importante el concepto de Kinch (3) quien sostiene que mientras más precoz es el estado clínico, más precoz el embarazo y más precoz el tratamiento, mejores resultados se consiguen. Barber y Brunschwig (11) dan un porcentaje global de curaciones del $56 \%$ para todas las edades y estados clínicos tratados únicamente con cirugía pero con mejor pronóstico para las intervenciones practicadas durante el embarazo que en el puerperio.

Jordan y Darnalt (12), en 1955 estudian 10 casos de Ca de cérvix y embarazo y son partidarios de la cirugía radical en los estados I y || diagnosticados precozmente, sin tener en cuenta la vida fetal sino la materna - esperar el máximo de viabilidad en la segunda mitad de la gestación para practicar Cesárea Corporal e Histerectomía Radical.

\section{Complicaciones de la Cirugía Radical}

Fístula uretero-vaginal: 2

Fístula uretero-vaginal - Eventración: 1

Absceso pararectal - Obstrucción intestinal: 1

O'Leary (14), presenta 5 casos de Cesárea seguida de histerectomía radical y uno con Histerectomía Abdominal y Cirugía Radical, en donde se presentaron una fístula uretero-vaginal y otra vésico-vaginal como complicaciones quirúrgicas.

\section{Resultado fetal}

Fetos vivos: 14

Fetos muertos: 4

Abortos: 17

Puérperas: 2

\section{Anatomía patológica}

En todos los casos en donde se practicó histerectomía radical, sola o asociada a cesárea corporal, se encontró Ca escamocelular infiltrante del cérvix (11) casos.

\section{Mortalidad}

Tres (3) casos.

Primer caso murió durante el acto quirúrgico de una histerectomía radical por anemia aguda, hipofibrinogenemia.

Otro caso tuvo su parto por las vías naturales y falleció a los 10 días del puerperio por diseminación carcinomatosa; y el otro caso se le practicó cesárea e histerectomía radical, presentó en el postoperatorio absceso pararectal, estrechez uretral e hidronefrosis derecha, obstrucción intestinal y falleció por una sepsis. 


\section{Controles}

Hasta 3 años

Hasta 5 años

Recientes

Sin control

$$
\begin{aligned}
& =\quad 2 \\
& =\quad 1 \\
& =\quad 3 \\
& =\quad 31 \\
& \hline 7
\end{aligned}
$$$$
\begin{array}{r}
6 \% \\
3 \% \\
9 \% \\
82 \% \\
\hline 100 \%
\end{array}
$$

Es criticable el gran número de enfermas que no volvieron a control $(82 \%)$; las que se controlaron hasta los cinco años (3\%), no volvieron después; todos sabemos que los controles de nuestras enfermas en nuestro medio es muy difícil. Creo, que todos debemos hacer algo por conseguir que no se sigan perdiendo tanto material humano, lo cual imposibilita hacer un buen seguimiento $y$ pronóstico de las mismas.

\section{Conclusiones}

1. Se analizan y estudian 37 casos de Carcinoma Invasivo de Cérvix concomitante con embarazo en un lapso de 11 años en el Instituto Materno Infantil "Concepción Villaveces de Acosta" del 1․ de Enero de 1960 al 30 de Septiembre de 1971.

2. La frecuencia de tal concomitancia es muy variada. En general es de un caso por cada 2.000 embarazos. En el IMI es de 0,031\%.

3. Todos los casos ocurrieron en mujeres multíparas; lo cual ha hecho suponer a varios autores sobre el papel importante que tiene los desgarros cervicales, las eversiones y laceraciones tan frecuentes en las multíparas.

4. La mayor incidencia ocurrió en las pacientes entre los 36 y los 45 años.

5. En cuanto al estado del cuello un gran número presentaban erosiones, ulceraciones, masas polipoides etc.
6. El motivo de consulta fue de flujo genital, hemorragias, dolor etc., más los propios del estado de gravidez.

7. La mayor frecuencia de carcinoma en la mujer gestante ocurrió entre la 36! y la 40: semana de gestación.

8. La mayor incidencia ocurrió en los estados II y I. Desafortunadamente nuestras pacientes llegan con un estado avanzado cuando el pronóstico es bastante incierto.

9. Lo criticable es la deficiencia en no haber tomado 27 citologías vaginales en estas pacientes; sin embargo, es bueno aclarar que en nuestra institución éste procedimiento solo se realiza de manera rutinaria desde hace seis años y la mayoría de los casos ocurrieron antes de este año, cuando la citología vaginal era un método de rara aplicación clínica. No hubo ningúnú caso de falso negativo.

10. En todos los casos en que se tomó biopsia fueron positivos para $\mathrm{Ca}$ in situ (1) y para Ca escamocelular infiltrante (36 casos).

11. Se presenta un cuadro de los tratamientos con su estado clínico correspondiente. Notamos que algunos casos se trataron erróneamente debido a conceptos diversos y no muy claros relacionados con el manejo de estas pacientes. Se dan las normas a seguir en estas clases de enfermas, normas que están de acuerdo con lo recomendado por la mayoría de los autores. También se critican dos casos con parto por las vías naturales ya que aparte de la hemorragia que pueda producirse durante la dilatación cervical, la diseminación cancerosa es lo más corriente disminuyendo por consiguiente el pronóstico de estas enfermas. 
12. Resultado fetal fue de 14 fetos vivos, 4 fetos muertos, 17 abortos $y$ 2 puerperas.

13. Hubo cuatro complicaciones de la cirugía radical: dos fístulas uretero-vaginal, una asociada a eventración y una paciente con un absceso pararectal y obstrucción intestinal.

14. Anatomía Patológica Final: en todos los casos en donde se practicó histerectomía radical, sola o asociada a cesárea corporal, se encontró carcinoma escamocelular invasivo en el cérvix.

15. En cuanto a la mortalidad hubo tres casos: uno ocurrió durante el acto quirúrgico, otro a los diez días del puerperio después de haber tenido el parto por las vías naturales posiblemente debido a una diseminación carcinomatosa y el otro se le practicó cesárea e histerectomía radical, habiendo presentado en el postoperatorio absceso pararrectal, estrechez ureteral e hidronefrosis derecha y obstrucción intestinal, falleciendo por una sepsis generalizada.

16. Los controles es de criticar pues la mayoría de las enfermas no volvieron a control y las pocas que se controlaron hasta por cinco años no volvieron.

Todos estamos de acuerdo lo difícil que es controlar estas enfermas gracias a idiosincrasia de nuestras pacientes.

\section{Summary}

The authors analyze 37 cases of invasive carcinoma of the cervix associated with pregnancy, with an IMI frequency of $0.031 \%$ all occurring in multipara women between 36 and 45 years of age.
A large number of these patients showed benign type cervical lesions (erosions, polypus, ulcerations) and consulted for genital flux, hemorrhage and pain, frequent symptoms in gravidity.

Twenty four patients (64\%) corresponded to clinical status $I$ and II, being frequent the cases of late consultation (13) which arrived to consultation in status III and IV.

The treatment procedure is recommended in agreement with the clinical status and the gestational age.

The results may be summarized as follows: two of the patients were puerperae. Four showed complications in radical surgery: two with ureterovaginal fistulae, associated with eventration; one with pararectal obscess and one with intestinal obstruction. Three of the patients died: one during surgery; another on the 10th. day of puerperium after normal birth as a cause of carcinomatous dissemination; and the third after radicals cesarian hysterectomy for pararectal abscess, hydronephrosis and generalized sepsis.

\section{BIBLIOGRAFIA}

1 MARSH, M. y FRITZGERAL P. J.: Carcinoma in situ of human uterine cervix in pregnancy; prevalence and post-pregnancy persistence. Cancer, 9: 11965, 1956.

2 KISTNER, R. W. y GORBACH, A. C.: Cervical cancer in pregnancy review of the literature with presentation of 30 edditional cases. Obst. \& Gynec. 9: 554. 1957.

$3 \mathrm{KINCH}, \mathrm{R}$. A.: Factors affecting the prognosis of cancer of the cervix in pregnancy. Am. J. Obst. Gynec. 82: 45, 1961.

4 LEON JUAN. Tratado de Obstetricia. Tomo II. Pág. 189. 1963.

5 EMGE, L. A. Influence of pregnancy on tumor growth. Am. J. Obst. \& Gynec. 28 : 682. 1934. 
6 WALDROP G. M. PALMER J. P.: Ca of the cervix associated with pregnancy. Am. J. Obst. \& Gynec. 86: 202, 1963.

7 WILSON, J. R.: Carcinoma of the cervix complicated by pregnancy, Am. J. Obst \& Gynec. 50: 275, 1945.

8 AREVALO EDO y LOZANO A. Embarazo y Malignidad. Rev. Obst. y Ginecología. Vol. XXVII № 1. Enero-Febrero de 1966.

9 GREENSPAN E. M. y LENNICK G. Embarazo y cáncer. Complicaciones Médicas, quirúrgicas y ginecológicas en el embarazo. Editorial Interamericana. 1967.
10 WAUGH, E. S. y PIKE, A. H.: Correlation of Papanicolaou smears and cervical cultures during pregnancy. Obst. \& Gynec. 9: 143, 1957.

11 BARBER H. R. K. BRUNSCHWING A.: Gynecologie cancer complicating. Pregnancy. Am. J. Obst. \& Gynec. 85: 156, 1963.

12 JORDAN S., DARNALT E.: Memorias de la Segunda Convención Col. de Obst. y Ginec. Nov. 29. Dic. 3 1955. Edición especial de la Revista Colombiana de Obstetricia y Ginecología. 